\title{
L'apprentissage des stratégies de mise en texte et de révision chez des élèves francophones en milieu minoritaire : une étude de cas contrastés
}

\author{
MARTINE CAVANAGH \\ Faculté Saint-Jean, Université de l'Alberta
}

SYLVIE BLAIN

Université de Moncton

\begin{abstract}
Résumé
L'objectif de cette étude est d'évaluer l'efficacité d'une séquence didactique adaptée à l'écriture d'un récit imaginaire et d'un récit réaliste sur la capacité d'élèves francoalbertains et néobrunswickois de neuf et dix ans à composer des textes narratifs cohérents. Pour ce faire, nous avons recueilli et analysé des données quantitatives et qualitatives. Dans le contexte de cet article, nous rapportons uniquement les résultats qualitatifs issus d'une étude de cas multiples s'insérant dans une recherche de plus grande envergure. Cette étude de cas a été conduite auprès de quatre élèves de niveaux d'habiletés variés en écriture que nous avons observés et interviewés en ce qui a trait à l'appropriation des stratégies de mise en texte et de révision. L'analyse des résultats révèle que tous les élèves sont plus conscients des critères d'un récit cohérent et qu'ils se basent sur ces critères pour évaluer leur texte et y apporter des modifications afin de l'améliorer. De plus, ils démontrent une capacité accrue à verbaliser leur démarche de mise en texte et de révision et à justifier les changements opérés. Par contre, les élèves plus forts se distinguent des élèves plus faibles dans la mesure où, quand ils rédigent et révisent, ils tiennent compte d'un nombre plus élevé de dimensions textuelles et utilisent davantage de métalangage lié au récit et au processus d'écriture pour évoquer leur démarche. L'analyse révèle également que les élèves plus faibles pourraient bénéficier de plus de soutien au moment de la mise en texte.
\end{abstract}

\section{Introduction et problématique}

En cette ère de l'information et des communications, la simple acquisition des connaissances n'est plus suffisante pour préparer les jeunes d'aujourd'hui au monde de demain. L'école vise donc davantage le développement des compétences (Alberta Learning 1998 ; Ministère de l'Éducation, 2013). Parmi ces compétences, mentionnons les communications orale et écrite efficaces ainsi que l'accès et l'analyse des informations (Rosefsky-Saavedra et Opfer, 2012), souvent qualifiées de surabondantes en raison de l'accès à Internet. Dans ce contexte, la compétence à écrire est particulièrement importante si l'on veut donner les outils nécessaires aux citoyens de demain pour qu'ils participent activement à la société démocratique. En effet, en apprenant à écrire, l'élève développe la capacité à communiquer ses idées de façon claire, précise et organisée, ce qui lui permettra en tant qu'adulte de participer aux débats et à la prise de décisions démocratique par rapport à des projets qui le concerne. Or, cette compétence demeure difficile à acquérir pour un grand nombre de 
francophones dans le monde ${ }^{1}$. Cela est d'autant plus vrai pour les élèves francophones canadiens vivant en contexte minoritaire, où l'anglais prédomine. En effet, ces élèves ont tendance à rédiger des textes courts et décousus, à utiliser des phrases simples, à mal enchaîner les idées entre les phrases et à laisser beaucoup d'idées implicites, ce qui nuit à la compréhension de leur message. Plusieurs raisons peuvent expliquer ces difficultés. Du côté des élèves, il existe une certaine insécurité linguistique (Boudreau et Dubois, 1992) qui entraine chez eux un manque de motivation à s'exprimer en français et donc un manque de pratique dans cette langue (Cavanagh et Blain, 2009). À cela s'ajoutent des difficultés sur le plan des stratégies cognitives qui sous-tendent la production d'un texte efficace (Conseil des ministres de l'Éducation du Canada, ou CMEC, 2004 et 2011). Du côté des enseignants, on note une tendance à recourir à une pédagogie traditionnelle axée sur le texte en tant que produit fini et sur la correction des erreurs plutôt que sur la démarche rédactionnelle qui sous-tend l'activité de construction de sens qui est au cœur de l'acte d'écrire (Gilbert, LeTouzé, Thériault et Landry, 2004 ; Cormier 2005 ; Cazabon, 2005). Cette situation s'explique, en partie du moins, par le fait que les enseignants manquent de ressources adaptées et de formation sur l'enseignement explicite des stratégies d'écriture (Gratton, Boudreau et Chiasson, 2014). Une formation sur ce type d'enseignement s'avère particulièrement important dans les milieux minoritaires, puisque l'école est souvent le seul lieu où l'élève est exposé au français.

Pour pallier cette lacune, certains chercheurs ont étudié, en s'intéressant à l'apprentissage de divers types de texte, les effets de séquences didactiques orientées vers l'enseignement explicite de stratégies d'écriture (Cavanagh, 2006, 2007a, 2008, 2009 ; Cavanagh et Langevin, 2010) sur la capacité des élèves à produire des textes cohérents. Ces tentatives comportaient toutefois plusieurs limites. D'abord, la durée limitée des interventions (maximum six semaines) n'a pas permis aux élèves de progresser dans toutes les dimensions de la cohérence (pour une explication de ces dimensions, voir le cadre conceptuel) ciblées par l'intervention mise en place. Seule la dimension macrostructurelle a fait l'objet de progrès statistiquement significatifs. Ensuite, la nature exclusivement quantitative des résultats obtenus n'a pas permis de saisir comment des élèves de niveaux d'habiletés variés en écriture s'appropriaient les stratégies d'écriture enseignées. Une telle limite nous a empêchées d'identifier des formes de soutien approprié aux élèves plus faibles. Pour combler ces lacunes, nous avons entrepris une recherche longitudinale auprès d'un groupe de 88 élèves francophones de $4^{\mathrm{e}}$ année du primaire que nous avons suivi l'année suivante en $5^{\mathrm{e}}$ année. Ces élèves provenaient de deux provinces canadiennes, soit l'Alberta (population de 3645257 habitants dont 2\% sont francophones) et le NouveauBrunswick (population de 751171 habitants dont 30\% sont francophones). Même si les communautés francophones de ces deux provinces sont distinctes d'un point de vue démographique, elles partagent néanmoins des défis communs en ce qui concerne l'enseignement et l'apprentissage du français. Parmi les principaux défis se trouvent la question de la valorisation de l'apprentissage du français dans un contexte linguistique qui ne le favorise pas et le manque de ressources pédagogiques appropriées pour le contexte.

Cette recherche, qui s'est arrêtée sur l'apprentissage de l'écriture de textes narratifs, comportait un volet quantitatif et qualitatif. Dans le cadre de cet article, nous

\footnotetext{
${ }^{1}$ Martine BETTI-CUSSO et al. (2007), «École primaire. Les clés de la réussite », Le Figaro, no 1402, p. 38-43; Guillaume MALAURIE et al. (2007), «Le scandale de l'illettrisme », dossier dans Le Nouvel Observateur, no 2235 , p. $7-17$.
} 
présentons uniquement les résultats du volet qualitatif issus d'une étude de cas multiples s'insérant dans la recherche qui est de plus grande envergure. Ces résultats sont liés à la question de recherche suivante: comment des élèves de niveau d'habiletés variés en écriture s'approprient-ils les stratégies de mise en texte et de révision enseignées dans le contexte de l'écriture d'un récit narratif? Dans les prochaines sections, avant de présenter les résultats et d'en discuter, nous précisons le cadre conceptuel et la méthodologie par l'entremise de laquelle nous opérationnalisons notre question de recherche. Nous concluons en donnant des pistes pour les futures recherches.

\section{Cadre conceptuel}

Notre intervention a été élaborée à partir de trois concepts clés. Pour déterminer les objectifs d'apprentissage de nos séquences didactiques, nous nous sommes inspirées des travaux sur la cohérence textuelle, l'expertise rédactionnelle et leur acquisition. Les moyens concrets à mettre en place pour atteindre ces objectifs ont été identifiés à partir des travaux qui ont mis en évidence l'importance du rôle de l'échafaudage pour amener l'élève à faire des apprentissages de haut niveau. À travers l'explication de ces concepts, nous décrirons en partie les approches préconisées dans nos séquences didactiques, celles-ci prenant en compte les particularités de l'enseignement et de l'apprentissage du français (Cazabon, 2005 ; Cormier, 2005) en milieu minoritaire.

\section{Le concept de cohérence textuelle en lien avec le récit}

Les travaux menés en grammaire du texte dans les années 1970 ont mis en lumière le fait que la qualité d'un texte dépend notamment de sa cohérence. Cette cohérence résulte de la capacité du scripteur à rédiger un texte qui possède une certaine unité, tant des points de vue situationnel, macrostructurel que microstructurel (Carter-Thomas, 2000). Les séquences didactiques expérimentées dans le cadre de cette étude ont donc été élaborées pour que les élèves puissent développer les compétences associées à ces trois dimensions de la cohérence lors de l'écriture d'un récit.

La cohérence situationnelle. La cohérence situationnelle concerne la relation entre les idées sélectionnées et la situation de communication dans laquelle le texte est produit. Plus spécifiquement, elle résulte de la capacité du scripteur à incorporer dans son récit des détails pertinents et intéressants en tenant compte des divers paramètres de la situation d'écriture, c'est-à-dire du destinataire, du thème, de l'intention d'écriture et de l'effet recherché (Apotheloz et Mieville, 1989; Tauveron, 1995). Parmi ces paramètres, il y a également la prise en compte du type (par exemple, le type narratif) et du genre de texte (par exemple, le conte ou le récit de sciencefiction). Dans le cas de la présente étude, les élèves ont dû produire deux genres narratifs : un récit imaginaire (en $4^{\mathrm{e}}$ année) et un récit réaliste (en $5^{\mathrm{e}}$ année). Pour définir le concept d'imaginaire, nous nous sommes appuyées sur la définition de Lammertyn (2000), qui considère l'imaginaire comme "produit et source de l'imagination, monde d'images, monde non réaliste, réservoir des productions humaines culturelles individuelles et collectives, exprimant les multiples interprétations du monde. » (p. 54). Comme son nom l'indique, le récit réaliste, quant à lui, devait privilégier le réel en évoquant des faits qui pourraient se produire dans la réalité. Ce genre de récit est plus exigeant à produire pour le scripteur qui doit, d'une part, trouver des manières vraisemblables de résoudre les problèmes qui surviennent 
et, d'autre part, s'assurer que le texte ne comporte pas de contradictions avec l'univers réaliste qu'il a créé.

La cohérence macrostructurelle. Liée à la notion de types de textes (Adam, 2005), la cohérence macrostructurelle concerne l'organisation du texte dans sa globalité. Elle découle de la capacité du scripteur à découper son texte en paragraphes distincts, selon une structure canonique. Dans le cas du récit, cette structure comprend une situation initiale, un élément déclencheur, des actions, un dénouement et une situation finale. Toutefois, pour qu'un récit soit globalement cohérent, la simple présence de ses parties ne suffit pas, il faut également que celles-ci soient sémantiquement reliées entre elles (Lammertyn, 2000 ; Crinon, 2006). Le personnage principal est d'ailleurs un élément qui joue un rôle unificateur important puisqu'il traverse l'ensemble de la séquence narrative. C'est pourquoi, dans notre intervention, nous avons accordé une place privilégiée au personnage principal allant même jusqu'à l'utiliser pour complexifier les apprentissages d'une année à l'autre. Ainsi, dans le cas de la séquence didactique visant la production d'un récit réaliste, nous avons sensibilisé les élèves de $5^{\mathrm{e}}$ année à l'importance d'établir un lien entre ce que le personnage principal est (son trait de personnalité dominant comme la curiosité) et ce qu'il fait dans l'histoire (les actions qu'il entreprend pour résoudre le problème auquel il est confronté) (Tauveron, 1995). Finalement, la cohérence globale exige que le scripteur assure une cohérence temporelle en utilisant, entre autres, des organisateurs textuels (par exemple, un jour, le lendemain, etc.) pour situer les actions dans le temps (Chartrand, Aubin, Blain et Simard, 2011).

La cohérence microstructurelle. La cohérence microstructurelle, ou cohérence « locale » (Charolles, 2006), se joue sur le plan de la phrase. Elle résulte d'abord de la capacité du scripteur à faire progresser son texte en ajoutant continuellement de nouvelles informations. Cette dimension de l'écriture présente des défis particuliers aux jeunes narrateurs. D'une part, ils négligent souvent de développer leurs idées à l'intérieur des différentes parties du récit en oubliant notamment de décrire les personnages et d'expliquer leurs buts, leurs motivations et la façon dont ils réussissent ou échouent dans leurs tentatives (Crinon, 2006). D'autre part, ils ont tendance à faire des digressions et à présenter dans l'action une trop grande quantité de dialogues inutiles, laissant le lecteur dans la confusion (Tauveron, 2009). La cohérence microstructurelle découle aussi de la capacité du scripteur à assurer la continuité des idées au fur et à mesure que son texte progresse (voir plus loin le processus de mise en texte). Ce type de cohérence implique donc le respect d'un équilibre entre la progression et la cohésion des informations dans le texte.

\section{Les processus et les stratégies qui sont à la base d'un texte cohérent}

Les travaux en psychologie cognitive portant sur le fonctionnement du scripteur novice et du scripteur expert ont montré que, chez le scripteur habile, la production d'un texte cohérent implique la mobilisation de processus cognitifs de haut niveau, dont l'activation passe par l'emploi de stratégies efficaces pouvant être ou non automatisées (Tardif, 1992). Dans le cas où une stratégie n'est pas automatisée, elle revêt alors un caractère conscient et peut se définir comme "un ensemble de décisions prises pour écrire un texte et qui débouchent sur une série d'actions coordonnées en tenant compte des composantes de la situation d'écriture » (Legendre, 2005 p. 1261). Les stratégies d'écriture sont généralement spécifiques au type de texte visé et elles sont réparties en fonction des trois processus cognitifs identifiés par 
Hayes et Flower (1980), soit la planification, la mise en texte et la révision qui sont composées de plusieurs sous-processus et stratégies. Étant donné le contexte bilingue de la recherche, il convient de préciser que les processus d'écriture sont les mêmes en langue première (L1) et en langue seconde (L2), sauf qu'en L2, les connaissances plus limitées de la langue cible peuvent avoir une incidence sur le parcours scriptural (Blain, 2003). Par exemple, la planification chez les scripteurs en L2 est généralement moins longue, la mise en texte est moins fluide et les pauses sont plus fréquentes. Dans ce qui suit, nous décrirons plus en détail les sous-processus et les stratégies de la mise en texte et de la révision qui sont au cœur de la présente étude.

Les sous-processus et les stratégies de la mise en texte. Au moment de la mise en texte, le scripteur habile passe d'une organisation schématique non séquentielle à une organisation linéaire puisque son travail consiste à transformer les bribes d'idées répertoriées dans son plan en phrases complètes qui se suivent (Fayol, 1997). Pour ce faire, il mobilise quatre sous-processus. Le premier consiste à développer ses idées clés en suivant l'ordre établi lors de la planification. Les trois prochains sousprocessus sont mobilisés conjointement avec le premier puisqu'au fur et à mesure que le scripteur développe les idées de son texte, il fait en sorte, en même temps, d'une part, que ses idées s'enchainent, en utilisant des procédés de reprise de l'information (pronom, synonyme, etc.), et, d'autre part, que les rapports entre les idées soient explicitement exprimés grâce à l'emploi de connecteurs. En plus des connecteurs spatiaux (par exemple derrière, devant, près de), qui servent à situer les éléments par rapport aux autres, Fayol (1996) distingue trois types de connecteurs : ceux qui marquent la chronologie d'évènements (puis, après, ensuite); ceux qui expriment le caractère inattendu d'une action (mais, tout à coup, soudain) et ceux qui évoquent une relation de causalité (ainsi, donc, alors). Enfin, pour faciliter la compréhension du lecteur, le scripteur habile marque la structure d'ensemble du texte en y insérant des organisateurs textuels (Romain, 2007). En somme, au moment de la mise en texte, les stratégies mobilisées par le scripteur habile prennent la forme d'un ensemble de procédés linguistiques appropriés au type de texte rédigé.

Les sous-processus et les stratégies de la révision. Au moment de la révision, le scripteur habile active deux sous-processus (Groupe EVA, 1996). Le premier consiste à évaluer la qualité de son texte tant du point de vue de la grammaire du texte que de celui de la grammaire de la phrase. Pour ce faire, il utilise la stratégie de relecture ciblée en relisant plusieurs fois son texte avec des critères précis en tête. Pour faciliter cette tâche, il peut se servir d'une grille de vérification, qui explicite les critères de qualité du texte, utiliser un code de révision ou recourir à des outils de référence. Il peut aussi demander à un pair de relire son texte et de lui signaler les sources d'incompréhension. Lorsque des faiblesses sont détectées, le scripteur mobilise le deuxième sous-processus, qui consiste à modifier ou à réécrire des parties de son texte à l'aide de stratégies prenant la forme de manipulation syntaxique telles que la suppression, l'ajout, le déplacement ou le remplacement de certains segments de phrase. Si le texte est rédigé sur papier, l'emploi de signes de révision (encoches, flèches, rayures, etc.) est une stratégie efficace qui lui permet d'apporter des changements directement dans son texte sans avoir à le réécrire en entier. 


\section{L'acquisition de la compétence rédactionnelle}

Pour savoir où se situe le scripteur novice sur les plans de son apprentissage des processus et des stratégies d'écriture, il est utile de comprendre comment s'acquiert la compétence rédactionnelle. Les travaux de Brassard (1998), basés en partie sur les recherches de Karmiloff-Smith (1992) sur le développement de l'oral chez les jeunes scripteurs, ont permis d'identifier trois phases d'acquisition de la compétence rédactionnelle. Durant la première phase, le novice travaille au niveau « micro » de la phrase, essentiellement la cohérence microstucturelle. Il est surtout préoccupé par les détails liés au thème sur lequel il doit rédiger. Ce traitement par données locales l'amène à écrire des textes-listes très décousus, voire incohérents. Pour faire un parallèle avec les travaux bien connus de Bereiter et Scardamalia (1987), cette phase correspondrait à la stratégie globale d'expression des connaissances (knowledge telling), qui caractérise le scripteur novice et qui consiste à énumérer tout ce que l'on sait sur le sujet selon un processus d'association d'idées.

La deuxième phase est marquée par l'apparition de la capacité à hiérarchiser les idées du texte en s'appuyant sur ses connaissances des schémas prototypiques intégrant ainsi la cohérence macrostructurelle. Durant cette phase, le scripteur s'adonne à un traitement par concepts, mais de façon mécanique, puisqu'il a tendance à remplir les cases vides du schéma textuel qu'il a en mémoire, mais sans tenir compte de la situation d'écriture. On observe souvent à cette étape une régression au niveau «micro », le texte étant moins riche en détails. Cette phase serait néanmoins une étape significative vers le développement de la compétence rédactionnelle, dans la mesure où le scripteur se montre conscient du fait que les informations qu'il est en train de gérer en écrivant n'appartiennent pas au même niveau de généralité, certaines étant plus englobantes que d'autres.

La troisième phase correspond à l'écriture du scripteur compétent. Elle se caractérise par la capacité du scripteur à gérer conjointement les dimensions « macro » et « micro » du texte en interaction constante avec les divers paramètres de la situation d'écriture, c'est-à-dire la cohérence situationnelle. Ce traitement souple par concept, qui serait à la base de la création d'un texte cohérent, correspondrait à la stratégie de transformation des connaissances (knowledge transforming) de Bereiter et Scardamalia (1987). Dans la lignée des recommandations de Brassard (1998), notre intervention a donc pour objectif ultime d'amener les élèves à dépasser la phase initiale de traitement par données locales en favorisant chez eux la construction de connaissances sur le schéma prototypique du récit, et ce, afin qu'ils s'en servent comme cadre d'action pour l'emploi et la gestion active et récursive des processus et des stratégies d'écriture en lien avec les différents niveaux textuels et la situation d'écriture. Toutefois, un tel objectif est complexe, surtout pour les élèves en contexte minoritaire, qui ont des limites langagières, et son atteinte nécessite l'offre d'un soutien approprié appelé échafaudage.

\section{L'échafaudage}

Les séquences didactiques que nous avons expérimentées exploitent plusieurs formes d'échafaudage (Vygotsky, 1978), ce concept correspondant à un soutien progressivement retiré selon les besoins particuliers des élèves. Parmi ces formes de soutien, le modelage, c'est-à-dire la verbalisation par l'enseignante de son raisonnement tandis qu'elle emploie une stratégie d'écriture devant les élèves en s'appuyant sur la fiche aide-mémoire qui la représente, occupe une place importante (Graham, 2006 ; Graham, Mckeown, Kiuhare et Harris, 2012). Ainsi, dans le cadre de notre intervention, le processus de mise en texte est soutenu par l'enseignante qui 
modélise l'emploi de la stratégie «Apporter avec moi». Cette stratégie est représentée visuellement par une valise contenant des exemples de procédés de reprise d'information (pronoms, synonymes, etc.). La métaphore de la valise rappelle à l'élève qu'à chaque fois qu'il ajoute une nouvelle phrase, il doit apporter avec lui une partie de la phrase précédente afin que ses idées s'enchainent. Utilisés conjointement avec des connecteurs, ces procédés de reprise d'information permettent d'assurer la continuité des idées d'une phrase à l'autre tout en exprimant les relations qui unissent les idées entre elles.

Le processus de la révision, quant à lui, est soutenu par l'emploi de deux stratégies représentées également sur des fiches aide-mémoire. La première stratégie intitulée «Mieux voir mes idées » est symbolisée par des lunettes contenant des questions associées à chaque partie du récit. Comme dans d'autres recherches (Blain, 2001 ; Blain et Lafontaine, 2010), cette stratégie est utilisée dans le cadre d'un travail en dyades où les élèves échangent leur texte et se posent mutuellement des questions afin d'amener leur partenaire à clarifier oralement ses idées. Par la suite, chaque scripteur est invité à recourir à divers signes (encoches, flèches, etc.) afin de modifier son texte (en faisant des déplacements, remplacements, ajouts ou suppressions) ${ }^{2}$ sans avoir à le recopier dans son entier. La seconde stratégie prend la forme d'une grille de vérification structurée autour des parties du récit qui encourage le scripteur à relire son texte de manière critique et à l'améliorer à la lumière des critères d'un récit cohérent qu'il aura construits au fil de sa participation aux diverses tâches prévues dans la séquence. Pour rappel, nous cherchons à répondre à la question suivante : comment des élèves de niveaux d'habiletés variés en écriture s'approprient-ils les stratégies de mise en texte et de révision enseignées dans le contexte de l'écriture d'un récit narratif?

\section{Méthodologie}

Pour l'ensemble de notre recherche, selon le paradigme pragmatique (Johnson et Onwuebbuzie, 2004), nous avons choisi une méthodologie mixte comportant un volet qualitatif et un volet quantitatif. Dans le cadre de cet article, nous présentons uniquement le volet qualitatif, qui se réalise sous la forme d'une étude de cas contrastés. Dans cette section, nous décrivons d'abord les participants ayant pris part à la recherche, puis l'intervention, avant de présenter le déroulement de la collecte et l'analyse des données qualitatives en lien avec les stratégies de mise en texte et de révision.

\section{Participants}

Quatre élèves de la $4^{\mathrm{e}}$ année en 2011-2012, que nous avons suivis l'année suivante en 2012-2013 alors qu'ils étaient en $5^{\mathrm{e}}$ année, ont participé à la recherche. Ils fréquentaient des écoles francophones du Nouveau-Brunswick et de l'Alberta. Les directions des deux districts francophones d'Edmonton et de Moncton ont donné leur assentiment initial au projet et elles ont laissé le choix aux directions d'écoles de participer à notre étude. Dans chacune des régions, Moncton et Edmonton, deux classes provenant de la même école ont accepté de faire partie de la recherche. Parmi ces quatre classes, nous avons choisi deux filles et deux garçons (une fille - un garçon dans chaque province) pour l'étude de cas. Dans chacune des provinces, il y avait un élève fort et un élève faible en écriture. Nous avons utilisé les

\footnotetext{
${ }^{2}$ Ces termes font référence à des manipulations syntaxiques mobilisées par le scripteur lors de la réécriture de son texte pour que le lecteur soit mieux en mesure de comprendre le message véhiculé.
} 
pseudonymes Annabelle (Alberta) et Alain (Nouveau-Brunswick) pour faire référence aux élèves forts et Bruno (Alberta) et Julie (Nouveau-Brunswick) pour faire référence aux élèves faibles. Le niveau d'habileté des élèves en écriture a été déterminé dès le début de l'étude à la suite d'un prétest que nous avons administré à tous les élèves. Pour ce prétest, les élèves devaient rédiger un récit imaginaire à partir d'un choix de deux situations d'écriture. Les textes produits par les élèves ont été évalués du point de vue des trois dimensions la cohérence textuelle à partir d'une grille élaborée par Cavanagh (2007b, p. 202-205). Selon les réponses obtenues aux questionnaires mesurant l'utilisation de l'anglais, du français ou d'autre langue parlée à la maison, seul Alain grandit dans une famille endogame, c'est-à-dire une famille où les deux parents sont francophones. Nos participants sont donc représentatifs des francophones en milieu minoritaire où la majorité grandit dans des familles exogames et parle les deux langues officielles du Canada.

\section{Intervention}

L'intervention a pris la forme de deux séquences didactiques composées de 13 leçons chacune. La première séquence, qui visait la production d'un récit imaginaire par les élèves de $4^{\mathrm{e}}$ année, était basée sur les outils publiés par Cavanagh (2007b). La seconde, qui ciblait l'écriture d'un récit réaliste par les mêmes élèves en $5^{\mathrm{e}}$ année l'année suivante, était fondée sur des outils non publiés que nous avions élaborés en suivant la même approche basée sur l'enseignement explicite des processus d'écriture à l'aide d'échafaudage. Les cinq premières leçons avaient pour objectif la construction de connaissances sur le schéma prototypique du récit en analysant des récits bien construits et d'autres moins bien construits ${ }^{3}$ à l'aide d'un organisateur graphique représentant visuellement la structure du texte. Par la suite, l'enseignante a consacré huit leçons à la rédaction d'un récit en modelant les stratégies d'écriture devant les élèves. Plus spécifiquement, elle a consacré cinq leçons aux stratégies de planification ${ }^{4}$, une leçon aux stratégies de mise en texte et deux leçons aux stratégies de révision. Après chaque séance de modelage, les élèves étaient invités à utiliser la stratégie dans le contexte de l'écriture de leur propre récit et recevaient de la rétroaction de la part de l'enseignante sur leur manière d'utiliser la stratégie. Dans le cadre de cet article, nous examinons les résultats en ce qui a trait aux leçons portant sur la mise en texte (stratégie apporter avec moi) et la révision (stratégie mieux voir mes idées utilisée conjointement avec une liste de signes de révision et une grille de relecture).

Avant la mise à l'essai de cette intervention, les participants à la recherche ont rédigé un premier texte. Par la suite, ils ont rédigé deux récits imaginaires pendant qu'ils étaient en $4^{\mathrm{e}}$ année et deux récits réalistes pendant qu'ils étaient en $5^{\mathrm{e}}$ année.

\section{Formation des enseignantes}

Les enseignantes ont reçu une formation avant et pendant l'intervention. Pour dispenser cette formation, nous nous sommes inspirées du modèle conçu par Beard El-Dinary et Schuder (1993) $)^{5}$. Avant l'intervention, les enseignantes ont reçu une

\footnotetext{
${ }^{3}$ La notion de texte bien construit est fondée ici sur des critères liés à la cohérence macrostructurelle (voir le cadre conceptuel).

${ }^{4}$ Les résultats par rapport aux stratégies de planification ont été publiés dans l'article suivant : Cavanagh et Blain S. (2015). Planning strategies for Writing: Their Effect on the Textual Coherence of Imaginative Stories Created by Francophone Pupils in New Brunswick and Alberta. The International Journal of Literacie, 21 (2), 1-16.

5 Selon le modèle de Beard El-Dinary et Schuder (1993) une formation pour l'enseignement des stratégies doit comprendre les cinq composantes clés suivantes : 1) une justification théorique, basée sur les recherches, exigeant de s'attarder à l'enseignement des stratégies ;2) une présentation des buts visés par le programme d'intervention ;
} 
formation d'une durée de deux jours de la part des chercheuses. Lors de cette formation, nous avons abordé 1) les principes d'apprentissage de base issus de la psychologie cognitive et du socioconstructivisme sur lesquels l'intervention a été fondée et 2) la structure de la séquence didactique. Pendant l'intervention, les enseignantes ont bénéficié d'échanges informels avec les deux chercheuses sur leur enseignement des leçons et ont reçu des documents d'appui prenant surtout la forme de scénarios de modelage des stratégies. Ces scénarios détaillaient les étapes à suivre et les questions à se poser au moment de l'emploi des stratégies.

\section{Collecte et analyse des données}

Pour répondre à notre question de recherche, qui porte sur la façon dont les élèves habiles en écriture et les élèves moins habiles s'approprient les stratégies de mise en texte et de révision que nous leur avions enseignées, nous avons mené des entrevues semi-dirigées auprès des quatre élèves retenus avant l'intervention, tandis qu'ils rédigeaient leur premier récit, et durant l'intervention, alors qu'ils rédigeaient les quatre autres récits. Chaque élève était muni d'un Smart Pen et d'un cahier tramé, une nouvelle technologie qui nous a permis d'enregistrer à l'ordinateur ce qu'ils écrivaient et ce qu'ils disaient durant les entretiens que nous avons effectués. Les questions que nous leur avons posées étaient basées sur le modèle de l'expertise rédactionnelle de Hayes (2004). Nous présentons ci-dessous quelques exemples de questions que nous avons posées en lien avec la mise en texte et la révision.

Questions en rapport avec la mise en texte

1. Comment vas-tu travailler avec ton plan pour écrire ton histoire ?

2. Est-ce que tu penses à de bonnes façons de dire des choses? (par exemple dans la description, dans les actions, quand tu passes d'une phrase à l'autre ?)

3. Est-ce qu'il y a des mots qui fonctionnent bien pour raconter une histoire ? Questions en rapport avec la révision

1. Maintenant que tu as écrit ton histoire, est-ce que tu vas la relire ? Pourquoi ?

2. As-tu apporté des changements à ton histoire ? Si oui, lesquels ? Peux-tu me montrer comment tu as fait?

3. Pourquoi as-tu fait ces changements ?

L'analyse des entrevues s'est effectuée en quatre étapes. Tout d'abord, nos assistants ont retranscrit les enregistrements audio des entrevues. Puis, afin de tracer l'évolution de chaque élève par rapport à la mise en texte et à la révision, des citations pertinentes en lien avec chacun des cinq textes produits ont été sélectionnées parmi les données brutes et regroupées dans un tableau divisé en fonction des questions posées. Par la suite, toujours pour chaque élève, les citations retenues ont été analysées et une synthèse des progrès réalisés au fil de l'intervention a été effectuée. Enfin, une comparaison des synthèses réalisées pour les élèves forts et les élèves faibles a permis de faire ressortir, dans leur manière de gérer les processus de mise en texte et de révision en amont et durant l'intervention, des ressemblances et des différences entre ces deux groupes. À chacune des étapes, les chercheuses ont comparé leurs analyses

\footnotetext{
3) une description de la structure du programme d'intervention ; 4) une présentation des concepts clés autour desquels s'articule le programme d'intervention et 5) des séances de modelage et de pratique guidée des leçons prévues dans le programme d'intervention.
} 
respectives. Lorsque celles-ci ne concordaient pas, une discussion avait lieu et des ajustements étaient faits pour refléter le consensus auquel elles étaient parvenues.

\section{Résultats}

Dans cette section, nous présentons d'abord les résultats pour l'appropriation des stratégies de mise en texte en contrastant les deux types de scripteurs, forts et faibles. Nous citons les élèves pour appuyer certains de nos propos. Ensuite, nous suivons la même procédure pour démontrer comment les deux types de scripteurs se sont approprié les stratégies de révision. Nous terminons avec une discussion de l'ensemble des résultats.

\section{Mise en texte}

Quand, avant notre intervention, nous avons demandé aux scripteurs habiles : «Comment vas-tu travailler avec ton plan pour développer tes idées ? », ils répondent qu'ils font un plan sommaire et l'utilisent comme aide-mémoire durant la rédaction du brouillon. Alain, par exemple, verbalise succinctement sa démarche de mise en texte : Je regarde mon plan pour voir [...] qu'est-ce que je vais mettre dans mon histoire.

Pendant la première année de notre intervention, ils font un plan plus détaillé, ils commencent à verbaliser leur démarche de mise en texte en expliquant comment ils se servent de leur plan comme aide-mémoire pour faire des phrases et ils utilisent le métalangage du récit (comme la situation initiale; l'élément déclencheur; le personnage principal et les actions). Lors de la deuxième année, nous remarquons les mêmes comportements, mais de façon encore plus précise et détaillée; en effet, ces élèves évoquent plusieurs fonctions du plan et leur emploi d'autres stratégies :
Je vais regarder mon plan et je vais regarder toutes mes idées, je vais regarder dans ma situation initiale, je vais regarder s'il y a des idées à propos de mon personnage principal, à propos de mon joueur, je vais mettre celles qui sont en rapport avec le personnage dans où est-ce que je suis supposé les écrire. Je vais aussi utiliser [les stratégies] " gonfler le portrait», et « examiner à la loupe » pour [déterminer] le lieu. (Alain)

En ce qui a trait aux scripteurs faibles, nous avons deux cas de figure. Avant notre intervention, Bruno fait un plan, mais il ne s'en sert pas pour rédiger et ne verbalise pas sa démarche de mise en texte. Quant à Julie, elle fait un plan, elle l'utilise comme aide-mémoire et elle verbalise succinctement sa démarche, sans en évoquer l'utilité : Je vais regarder ce que j'ai écrit, donc, là, je suis rendue au numéro et je regarde mon numéro un. Je trouve ça [un plan] utile parce que sinon, je ne sais pas quoi faire et $j$ 'oublie toutes mes idées.

Lors des textes rédigés pendant notre intervention, nous notons des changements pour ces deux scripteurs faibles. Bruno fait un plan à l'aide du schéma, il s'en sert et il verbalise succinctement sa démarche en évoquant les détails de son plan qu'il a modifié, mais sans utiliser le métalangage du récit. Julie, de son côté, fait un plan à l'aide du schéma, elle verbalise sa démarche de façon plus détaillée en expliquant la différence entre le plan et le brouillon, et en insistant sur l'utilité du plan. Elle utilise le métalangage du récit :

La première phrase qu'on écrit, c'est la phrase la plus difficile.

J'ai décidé de commencer avec le nom [du personnage]. La 
grande différence entre le plan et l'histoire, c'est les mots-clés, les étapes..., mais l'histoire, c'est les détails et la cohérence.

J'utilise beaucoup mon plan pour le brouillon, sinon je ne serais pas où j'en serais.

En examinant comment les scripteurs habiles verbalisent la façon dont ils parlent de l'enchainement des phrases, des relations logiques et de la structure du texte, nous remarquons qu'avant notre intervention, ils n'évoquent pas de critères à respecter lors de la mise en texte et ils s'attardent à la ponctuation et aux erreurs d'orthographe lexicale et grammaticale. Pendant notre intervention, Alain évoque la syntaxe, les phrases complètes et pas trop longues, les pronoms pour remplacer les noms et la structure d'ensemble. Annabelle évoque les mêmes aspects en ajoutant l'importance de rester dans le sujet, les besoins du lecteur et les connecteurs de temps :

Quand je passe d'une phrase à l'autre, il faut continuer
graduellement pour ne pas changer le sujet. Je parle de mon
sujet de manière constante, c'est moins confusant [sic] pour le
lecteur. Le mot « soudainement», c'est que ça se passe tout de
suite. À la place de dire: "Dans deux secondes, quelque chose
arrive. »Il y a aussi «plus tard» et «finalement» [qui
permettent de voir] quand les actions vont se passer.

Nous n'avons pas trouvé le même type d'évolution chez nos scripteurs faibles. En effet, avant et pendant notre intervention, ils verbalisent cet aspect de la mise en texte de la même façon, c'est-à-dire qu'ils n'évoquent pas de critères à respecter lors de la mise en texte. Ils sont surtout préoccupés par la stratégie trouver les bons mots en français et la génération d'idées en lien avec les différentes parties de leur histoire.

En somme, exception faite du sous-processus « enchainement des phrases, des relations logiques et de la structure du texte » pour nos scripteurs faibles, il semble donc que tous les scripteurs ont appris à mieux gérer l'ensemble des stratégies de mise en texte pendant notre intervention. Tournons-nous maintenant vers l'analyse de l'appropriation des stratégies de révision.

\section{Révision}

Une des stratégies de révision à développer comme scripteur est la capacité de se relire pour identifier des faiblesses, pour faire des changements et les justifier. Avant notre intervention, quand nous demandions aux participants : "As-tu apporté des changements à ton récit? Lesquels ? Peux-tu m'expliquer comment tu as fait ?», nous avons trouvé que les scripteurs forts font peu de changements, qu'il s'agit surtout de remplacements de mots et qu'ils sont rarement capables de justifier les changements :

J'ai supprimé [...] pour raccourcir mon histoire.

Chercheur: As-tu fait d'autres changements?

Annabelle: Non, pas vraiment. Juste des petits. Comme ici j'ai

changé : "Isaac se cachait dessous son lit », mais ici, c'est : «

Il se cachait en dessous du sofa.» 
L'analyse des entrevues menées pendant notre intervention révèle que ces mêmes scripteurs font plus de changements et que ces modifications sont de nature différente (remplacements, déplacements, ajouts) et à plusieurs niveaux (mot, phrase, texte). Ils utilisent plusieurs signes de révision (flèches, encoches, etc.). Ils prennent en compte la rétroaction de leurs pairs et ils justifient les changements en pensant à plusieurs critères (cohérence d'ensemble, cohérence interne du personnage, clarté, besoin du lecteur) :
J'ai ajouté comment elle se sentait. Depuis la mort de son oncle, elle n'est pas de bonne humeur et elle est toujours fatiguée et cela aura un rapport avec ses hallucinations plus tard. Puisqu'elle est fatiguée, ça va être plus cohérent si elle fait des hallucinations. (Annabelle)
Le changement a été fait, car une personne a lu mon texte et ne comprenait pas ce que je disais. J'ai fait un déplacement pour ajouter de la clarté. (Alain)

Tout comme leurs pairs plus habiles, les scripteurs faibles font très peu de changements avant notre intervention et ces changements ont comme objectifs de corriger leurs erreurs d'orthographe ou d'éviter la répétition.
J'ai barré, parce que je faisais des erreurs ou j'avais mal écrit une lettre. Parce que je disais [ce mot] deux fois dans mon histoire. (Bruno)

Pendant notre intervention, nous avons trouvé que ces mêmes scripteurs font plus de changements et qu'ils sont davantage préoccupés par le sens. Ils sont maintenant capables de justifier leurs changements en pensant au sens (clarté et précision) et en utilisant plus de métalangage :

J'ai déplacé " proche dans son quartier » au début parce que ça sonne mieux. C'est mieux au début, c'est plus clair. (Bruno) J'ai ajouté "bien sûr » pour que ça ait un peu plus de sens. (Bruno)

\section{Il a passé une nuit « glaciale ». Je trouvais que l'adjectif apportait une précision importante à l'histoire. (Julie)}

Selon l'ensemble des résultats obtenus pour les stratégies de révision, il semble donc que les deux types de scripteurs ont développé, à des degrés variables, leur capacité à verbaliser des stratégies de révision de plus haut niveau pendant notre intervention. Dans notre discussion, nous mettons en relation l'ensemble de ces résultats (appropriation des stratégies de mise en texte et de révision) avec des travaux sur l'acquisition de la compétence réactionnelle.

\section{Discussion}

Le scripteur novice, selon les travaux de Brassard (1998) et de KarmiloffSmith (1992), se concentre surtout au niveau « micro » de la phrase. Les quatre jeunes participants à notre recherche se situent tous à ce niveau avant notre intervention. En effet, pendant la mise en texte, ils s'attardent à la ponctuation et aux erreurs de surface 
ou «contraintes locales» (Hayes, 2004). Lors du processus de révision, ils se préoccupent de corriger les erreurs d'orthographe lexicale et grammaticale. Ils sont donc généralement préoccupés par les détails liés au thème sur lequel ils doivent rédiger ce qui correspond à l'expression des connaissances (knowledge telling) selon Bereiter et Scardamalia (1987).

Pendant notre intervention, nous remarquons l'apparition de l'habileté à se préoccuper de la structure du texte, le niveau « macro », tout en gardant un fort intérêt pour le niveau « micro » surtout chez les scripteurs plus habiles. Cela correspond à la deuxième phase de l'acquisition de la compétence rédactionnelle (Brassard, 1998). Plus spécifiquement, les scripteurs forts verbalisent leur démarche de mise en texte en évoquant la syntaxe, les phrases complètes, les pronoms pour remplacer les noms, l'importance de rester dans le sujet et les connecteurs de temps. Les scripteurs faibles demeurent plutôt au niveau «micro » lors de cette phase du processus rédactionnel, même pendant notre intervention. En revanche, pour la phase de révision, les deux types de scripteurs justifient leurs changements au niveau « macro»: cohérence d'ensemble, cohérence interne du personnage et clarté de l'ensemble du texte. Les scripteurs plus forts utilisent même le métalangage de façon systématique. Nous croyons que l'utilisation du schéma prototypique du récit est en partie responsable de cette prise en compte des contraintes plus globales du processus rédactionnel. Nous postulons que l'étayage constant a également joué un rôle dans ce passage de scripteur novice à intermédiaire.

La troisième phase correspond à l'écriture du scripteur compétent. Lors de cette phase, le scripteur devrait être capable de gérer les niveaux « micro » et « macro » tout en prenant en compte divers paramètres de la situation d'écriture, ce que Bereiter et Scardamalia (1987) nomment la transformation des connaissances (knowledge transforming). D'emblée, on pourrait croire qu'il ne soit pas réaliste de s'attendre à un tel niveau de développement chez des élèves du primaire. Or, certains scripteurs forts participant à notre étude de cas ont manifesté à certaines occasions cette habileté de prendre en compte les paramètres de la situation d'écriture tout en verbalisant la prise en compte des contraintes locales et globales. Annabelle, par exemple, mentionne à quelques reprises les besoins du lecteur. Cette phase demeure cependant en émergence, mais nous croyons que notre intervention, qui s'est étalée sur 16 mois, a permis à Annabelle de passer de scriptrice novice à l'émergence d'une scriptrice compétente.

\section{Conclusion}

L'objectif de cette étude de cas contrastée était de vérifier l'acquisition des stratégies de mise en texte et de révision chez des élèves francophones en milieu minoritaire canadien dans le cadre d'un enseignement explicite des stratégies d'écriture visant la cohérence textuelle. Les quatre participants de notre étude étaient des élèves de $4^{\mathrm{e}}$ année au début de l'intervention qui s'est poursuivie alors qu'ils étaient en $5^{\mathrm{e}}$ année.

Selon les analyses issues des entrevues que ces élèves nous ont accordées alors qu'ils ont rédigé cinq récits différents, dont un avant notre intervention, tous les élèves semblent avoir développé des capacités métacognitives, passant de la phase du scripteur novice à intermédiaire (Brassard, 1998). Tant les deux scripteurs forts que les deux scripteurs moins habiles sont plus conscients des critères d'un texte cohérent et ils ont été capables de se baser sur ces critères pour évaluer leur texte. Ils prennent en compte non seulement la cohérence microstructurelle, mais aussi macrostructurelle. Ils démontrent également une capacité accrue à verbaliser leur 
démarche et à justifier les changements opérés en utilisant le métalangage. Les plus forts tiennent compte d'un plus grand nombre de dimensions que les plus faibles et ils s'approprient davantage de métalangage. Une de nos participantes a même été capable de verbaliser l'importance de la prise en compte des besoins du lecteur ce qui se rapproche de la compétence à écrire puisqu'elle se préoccupe aussi de la cohérence situationnelle.

Les scripteurs moins habiles auraient besoin de plus de soutien et de plus de temps pour la mise en texte puisque la prise en compte à la fois des contraintes locales et globales semblait encore difficile pour eux. En particulier, nous croyons que la stratégie «Apporter avec moi », qui visait à aider les élèves à enchainer leurs phrases en leur suggérant des procédés de reprise de l'information (pronoms, synonymes, etc.), doit être revue, car la métaphore qu'elle mobilise s'avère très abstraite pour les élèves plus faibles. Elle devrait sans doute être remplacée par une stratégie qui aurait deux caractéristiques : 1) son appellation ferait directement référence à l'opération qu'elle permet de réaliser et 2) elle expliciterait les étapes de la marche à suivre afin de bien enchainer ses phrases. De plus, nous pensons que l'emploi de cette nouvelle stratégie devrait être précédé par quelques activités de lecture de paragraphes extraits de récits déjà analysés. Ces activités auraient pour but d'amener les élèves à repérer les divers procédés de reprise de l'information auxquels l'auteur a eu recours et à prendre conscience de leur importance pour assurer la continuité des idées d'une phrase à l'autre.

\section{Limites de notre étude}

Le nombre limité des cas étudiés ne nous permet pas de généraliser les résultats. De plus, en posant des questions à ces enfants, nous les amenions à réfléchir davantage à leurs stratégies, ce qui a pu biaiser les résultats. La désirabilité sociale est sans doute un autre facteur qui a pu contaminer nos données. Dans le cadre de cette étude de cas contrastée, nous n'avons pas vérifié la qualité des textes écrits par ces quatre élèves en particulier. Les résultats obtenus pour l'évaluation des textes produits par l'ensemble des participants à notre étude paraitront bientôt. ${ }^{6}$

Malgré ces limites, nous croyons que notre intervention axée sur un enseignement explicite des stratégies d'écriture a contribué à amener les élèves à dépasser la phase initiale de traitement par données locales en favorisant chez eux la construction de connaissances sur le schéma prototypique du récit. L'échafaudage a aussi permis une gestion active et récursive des processus et des stratégies de mise en texte et de révision en lien avec les trois types de cohérence textuelle, c'est-à-dire microstructurelle, macrostructurelle et, dans une moindre mesure, situationnelle.

\footnotetext{
${ }^{6}$ Nous avons mesuré la cohérence macrostructurelle, microstrucurelle et situationnelle de trois récits imaginaires rédigés en $4^{\mathrm{e}}$ année et de deux récits réalistes rédigés en $5^{\mathrm{e}}$ année pour les 33 élèves du groupe expérimental et les 41 élèves du groupe témoin. Nous avons évalué deux versions de chaque récit, c'est-à-dire le premier brouillon et la copie définitive. Les analyses de variance de type mixte inter et intra groupes indiquent que les deux groupes ont réussi à améliorer de façon significative leur cohérence textuelle dans les trois dimensions, mais que l'amélioration du groupe expérimental est significativement meilleure que celle du groupe témoin surtout pour les récits réalistes écrits lors de la $2^{\mathrm{e}}$ année de l'expérimentation (Blain et Cavanagh, à paraitre).
} 


\section{Pistes de recherches futures}

Les résultats de cette recherche menée dans le contexte de la production d'un récit nous amènent à recommander que d'autres études de cas soient menées dans d'autres genres de texte (argumentatif, explicatif, etc.) non seulement au primaire, mais aussi au secondaire. Nous proposons aussi d'explorer l'expérience des enseignants qui accordent une place importante aux stratégies d'écriture pour mieux comprendre les défis auxquels ils font face et identifier leurs besoins en matière de formation continue. Finalement, il nous semble nécessaire de mener des recherches en formation initiale pour savoir comment préparer efficacement les futurs enseignants à l'enseignement explicite des stratégies de lecture et d'écriture dans toutes les matières.

\section{Références}

Adam, J.-M. (2005). Les textes : types et prototypes. Récit, description, argumentation, explication et dialogue ( $2^{\mathrm{e}}$ éd.). Paris, France: Armand Colin.

Alberta Learning (1998). Programme d'études de français - langue maternelle. Edmonton, Alberta : Alberta Resources Branch.

Apotheloz, D., Mieville, D. \& Grize, J.-L. (1989). Cohérence et discours Argumenté, in M. Charolles (Eds.) The Resolution of Discourse Processing. Coherence or Consistency Dissonances. p. 68-87). Hamburg, Helmut Buske Verlag.

Beard El-Dinary, P., \& Schuder T. (1993). Seven Teachers Acceptance of Transactional Strategies Instruction during Their First Year Using It. The Elementary School Journal, 94(2), 207-219.

Bereiter, C., \& Scardamalia, M. (1987). The psychology of written composition. Hillsdale, NJ: Erlbaum.

Blain, S. (2001). Study of Verbal Peer Feedback on the Improvement of the Quality of Writing and the Transfer of Knowledge in Francophone. Language, culture and curriculum, 14(2), 156-170.

Blain, S. (2003). L'enseignement de l'écriture en milieu minoritaire canadien : problématique particulière et complémentarité des cadres théoriques en L1 et L2, in J.-M. Defays, B. Delcominette, J.-L. Dumortier et V. Louis (Eds.), Langue et communication en classe de français : convergences didactiques en langue maternelle, langue seconde et langue étrangère (pp. 185-200). Fernelmont, Belgique : Éditions Modulaires Européennes.

Blain, S., \& Cavanagh M. (À paraitre). Étude longitudinale mesurant les effets de l'enseignement explicite des stratégies rédactionnelles sur la cohérence des récits écrits par des élèves francophones vivant en milieu minoritaire. Éducation francophone en milieu minoritaire.

Blain, S., \& Lafontaine L. (2010). Mettre les pairs à contribution lors du processus d'écriture : une analyse des impacts du groupe de révision rédactionnelle sur le transfert des connaissances en orthographe grammaticale dans différents contextes linguistiques. Revue des sciences de l'éducation, 36(2), 469-491.

Boudreau, A., \& Dubois, L. (1992). Insécurité linguistique et diglossie: Étude comparative de deux régions de l'Acadie du Nouveau-Brunswick. Revue de l'Université de Moncton, 25 (1-2), 3-32.

Brassard, D. (1998). Approches cognitives de la didactique de la composition écrite, Psychologie et Éducation, 33, 13-29. 
Carter-Thomas, S. (2000). La cohérence textuelle. Pour une nouvelle pédagogie de l'écrit. Paris, France: L'Harmattan.

Cavanagh, M. (2006). Validation d'un programme d'intervention pour la cohérence des écrits argumentatifs au primaire. Revue des sciences de l'éducation, 32(1), 159-182.

Cavanagh, M. (2007a). Profil scriptural d'élèves du primaire en vue d'une intervention en milieu francophone minoritaire. Canadian Journal of Education/Revue canadienne de l'éducation, 30 (3) 691-724.

Cavanagh, M. (2007b). Stratégies pour écrire un récit imaginaire. Montréal, Québec: Chenelière Éducation.

Cavanagh, M. (2008). Vers un programme d'intervention pour aider les élèves francophones de 9-10 ans en milieu minoritaire à rédiger un récit cohérent. Éducation francophone en milieu minoritaire, 3 (1) 1-14.

Cavanagh, M., \& Blain, S. (2009). Relever quatre défis de l'enseignement de l'écrit en milieu francophone minoritaire. Les Cahiers franco-canadiens de l'Ouest, 21, 151-178.

Cavanagh, M., \& Langevin, R. (2010). The Quality of French Minority Students' Fictional Texts: A Study on the Influence of a Preferential Cognitive Style and Writing Strategy Scaffolding. Language, Culture and Curriculum, 23, 71-87.

Cazabon, B. (2005). Pour un enseignement réussi du français langue maternelle: fondements et pratiques en didactique du français. Sudbury, Ontario : Prise de parole.

Charolles, M. (2006). De la cohérence à la cohésion du discours, in F. Calas (Eds.), Cohérence et discours (pp. 25-38). Paris, France: Presses de l'Université de Paris-Sorbonne.

Chartrand, S.-G., Aubin, D., Blain, R., \& Simard, C. (2011). Grammaire pédagogique $d u$ français d'aujourd'hui (2 ${ }^{e} e ́ d$.). Montréal, Québec: Chenelière Éducation.

Conseil des ministres de l'Éducation du Canada (CMEC). (2011). PPCE de 2010 : Rapport de l'évaluation pancanadienne en mathématiques, en sciences et en lecture. Conseil des ministres de l'éducation du Canada, Toronto, Canada.

Conseil des ministres de l'Éducation du Canada (CMEC). (2004). Résultats pancanadiens des élèves francophones en milieu minoritaire au Programme d'indicateurs du rendement scolaire (PIRS). Rapport analytique. Toronto, Ontario : CMEC. Repéré à http://204.225.6.243/else/francophone/analysis.FR.pdf

Cormier, M. (2005). La pédagogie en milieu minoritaire francophone : une recension des écrits. Ottawa, ON: Fédération canadienne des enseignants et des enseignantes.

Crinon, (2006). Lire et écrire la fiction : quelques malentendus. Repères, 33, 61-79.

Fayol, M. (1996). La production d'écrits narratifs : Approche de psycholinguistique textuelle chez l'enfant et l'adulte in J. David \& S. Plane (Eds.), L'apprentissage de l'écriture de l'école au collège (pp. 9-36). Paris, France : Presses universitaires de France.

Fayol, M. (1997). Des idées au texte. Psychologie cognitive de la production verbale, orale et écrite. Paris, France: Presses universitaires de France.

Gilbert, A., Letouzé S., Thériault, J-Y., \& Landry R. (2004). Le personnel enseignant face aux défis de l'enseignement en milieu minoritaire francophone : Rapport final de recherche. Ottawa, ON: Fédération canadienne des enseignants et des enseignantes. 
Graham, S. (2006). Strategy instruction and the teaching of writing: A meta-analysis, in C.A. MacArthur, S. Graham \& J. Fitzgerald (Eds.), Handbook of writing research (pp. 187-207). New-York, NY : Guilford Press.

Graham, S., Mckeown, D., Kiuhare, S., \& Harris, K. R. (2012). A meta-analysis of writing instruction for students in the elementary grades. Journal of Educational Psychology, 104(4), 879-896.

Gratton, L., Boudreau R., \& Chiasson M. (2014). Enjeux de l'enseignement en contexte minoritaire francophone : Résultats de deux enquêtes pancanadiennes réalisées auprès du personnel enseignant. en milieu minoritaire: Synthèse de l'enquête. Ottawa, Ontario: Fédération canadienne des enseignants et enseignantes.

Groupe EVA (1996). De l'évaluation à la réécriture - Réécrire au cycle III. Paris, France: Hachette-INRP.

Hayes, J. (2004). What triggers revision? In L. Allal., L. Chanqouy \& P. Largy (dir.), Volume 13, Revision: Cognitive and instructional processes (pp. 9-20). Boston: Kluwer.

Johnson, R. B., \& Onwuegbuzie, A. (2004). Mixed methods research: A research paradigm whose time has come. Educational Researcher, 33(7), 14-26.

Karmiloff-Smith, A. (1992). Beyond Modularity, A Developmental Perspective of Cognitive Science, Cambridge, Massachusetts: The MIT Press.

Lammertyn, P. (2000). La sollicitation de l'imaginaire dans l'écriture des récits : intérêts et problèmes. Repères, 21, 53-77.

Legendre, R. (2005). Dictionnaire actuel de l'éducation (3e éd.). Montréal, Québec : Guérin.

Ministère de l'Éducation et du Développement de la petite enfance. (2013).

Programme d'études : Français 4-8. Fredericton, Nouveau-Brunswick :

Direction des programmes d'études et de l'apprentissage.

Romain, C. (2007). L'emploi des temps et des organisateurs textuels dans des textes narratifs d'élèves de 9 à 14 ans issus de milieux socioculturels contrastés. Revue des sciences de l'éducation, 33 (1), 209-235.

Rosefsky-Saavedra, A., \& Opfer, V.D. (2012). Learning 21st-century Skills Requires 21st-century Teaching. Phi Delta Kappa International, 94 (2), 8-13.

Tardif, J. (1992). Pour un enseignement stratégique : l'apport de la psychologie cognitive. Montréal : Éditions logiques.

Tauveron, C. (1995). Le personnage. Une clef pour la didactique du récit à l'école élémentaire. Neuchâtel-Paris, France: Delachaux et Niestlé.

Vygotsky, L. (1978). Mind in Society: The Development of Higher Psychological Processes. Cambridge (MA): Havard.

\section{Biographies des auteurs}

Martine Cavanagh est professeure titulaire à la Faculté Saint-Jean de l'Université de l'Alberta. Ses travaux de recherche, menés dans une perspective de psychologie cognitive, portent principalement sur l'enseignement de l'écriture de divers genres de texte à des élèves scolarisés dans des provinces en dehors du Québec où le français est la langue de la minorité. Elle s'intéresse aussi à la formation initiale des enseignants destinés à œuvrer dans les programmes d'immersion française et dans les écoles francophones en milieu minoritaire. 
Sylvie Blain est professeure titulaire à la Faculté des sciences de l'éducation de l'Université de Moncton. Elle a obtenu son doctorat en didactique des langues secondes en 1997 (Montréal) après avoir enseigné au Québec et en ColombieBritannique dans les écoles primaires. Elle donne les cours de didactique du français au baccalauréat et le cours "Stratégies cognitives » à la maitrise. Ses projets de recherche portent sur l'apprentissage de la lecture, de l'écriture en milieu francophone minoritaire ainsi que sur de l'intégration pédagogique des technologies. 